$\mathbb{T}$ periodica polytechnica

\author{
Civil Engineering \\ 58/4 (2014) 423430 \\ doi: $10.3311 /$ PPci.7856 \\ http://periodicapolytechnica.org/ci \\ Creative Commons Attribution (1) \\ RESEARCH ARTICLE \\ Bence Balogh / János Lógó \\ Received 2014-09-15, revised 2014-11-02, accepted 2014-11-12
}

\section{Optimal design of curved folded plates}

\begin{abstract}
The plated structures are one of the most frequently used engineering structures. The object of this research work is the optimal design of curved folded plates. This work is an ongoing investigation. There are various solution methods to analyze this type of structures. Here the finite strip method is used. At first single load condition is considered, but later the multiple load conditions are used for the design. The base formulation is a minimum volume design with displacement constraint what is represented by the compliance. For the multiple loading two equivalent topology optimization algorithms can be elaborated: minimization of the maximum strain energy with respect to a given volume or minimization of the volume of the structure subjected to displacement constraints. The numerical procedures are based on iterative formulas which is formed by the use of the first order optimality condition of the Lagrangian-functions. The application is illustrated by numerical examples.
\end{abstract}

\section{Keywords}

optimization - multiple loading $\cdot$ curved plate $\cdot$ curved folded plate $\cdot$ optimal layout $\cdot$ optimality criteria method $\cdot$ optimal design

\section{Bence Balogh}

Department of Structural Mechanics, Budapest University of Technology and Economics, Múegyetem rkp. 3, H-1111 Budapest, Hungary

e-mail: bbence@eik.bme.hu

\section{János Lógó}

Department of Structural Engineering, Budapest University of Technology and Economics, Múegyetem rkp. 3, H-1111 Budapest, Hungary

e-mail: logo@ep-mech.me.bme.hu

\section{Introductions}

The minimum weight design as an objective was a rather popular topic during "golden ages" of the optimization. The classical solutions of the different type of plate or shell problems can be followed by the works of Mroz [13], Prager \& Shield [14], Shield [17] from the end of 50-s of the last century. The design was elaborated in elastic or plastic ways. A good overview can be obtained by reading the report Rozvany et al. [16]. The design methods generally elaborated on deterministic based data but later it was extended to stochastic ones (e.g. Lógó [12]).

The optimal limit state design of prestressed thin-walled folded plate structures under multiple loading conditions was presented by Bergamini \& Biondini [2]. Lellep [6], Lellep \& Paltsepp [7,8] formed the optimal design formulation for inelastic shells included internally stiffened and/or supported ones.

Leng, Guest and Schafer [9] presented a comprehensive study on shape optimization of cold-form steel columns. Their optimal shape of the cold-formed steel lipped channel has doubled capacities than the conventional one. Gilbert et.al. [4] elaborated a genetic algorithm for optimisation of section capacity for thin-walled profiles.

Considering the solution methods, the optimality criteria as a tool for optimal design started its carrier from the beginning of 70-s of the last century (Gellatly \& Berke [3]). This is date when Rossow \& Taylor [15] published the very first topology optimization paper.

Here an optimality criteria based (Lógó [10]) design algorithm is combined by the finite strip formulations for curved folded plate design. The elaborated method is generally applied for single load condition but it can be extended for multiple ones, too. The extended formulations are presented, but the algorithm is derived for single load case. This paper is an extended version of the conference presentation of Balogh and Lógó [1].

\section{Formulation of the finite strip method for curved folded plates}

To be able to apply the finite strip for curved folded plate structures it is necessary to reformulate the general expressions. 

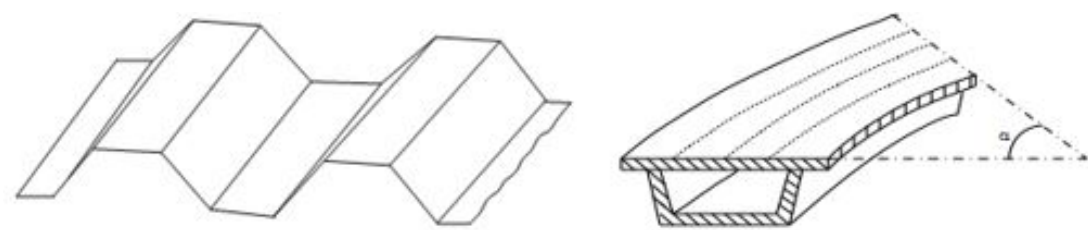

Fig. 1. A folded plate (left) and a curved box girder bridge (right)

Here the most general case of curved folded plates will be considered.

It has to note that the phrases "curved plate" and "shell" is identical as they are both plates with non-zero curvature. The phrase "folded" means that the joining elements at the nodal lines do not meet with the same tangent. One example for both folded plate and curved folded plate can be seen in Fig. 1

\subsection{Assumptions}

The shells are following first order shear deformation theory, as linear displacement variation is assumed through the thickness. Therefore the assumptions of the Mindlin-Reissner plate theory holds. This allows to take into account the effects of a constant transverse shear stress state by removing the normality conditions from the kinematic assumptions of classical plate theory. This way of approximation provides good results even under the side to thickness ratio of 20.

\subsection{Strains and displacements}

The displacement field at any point of the plate can be expressed as:

$$
\begin{gathered}
u(s, \theta, n)=u_{0}(s, \theta)+n \vartheta_{s}(s, \theta), \\
v(s, \theta, n)=v_{0}(s, \theta)+n \vartheta_{t}(s, \theta), \\
w(s, \theta, n) \cong w_{0}(s, \theta),
\end{gathered}
$$

where $u, v$ and $w$ are the displacements of a typical point in the $s, t$ and $n$ directions, $\vartheta_{s}$ and $\vartheta_{t}$ are the normal rotations contained in planes $s n$ and $t n$, while $\theta$ without a lower index stands for the radial coordinate in the cylindrical coordinate system. The sign convention is shown on Fig. 2. We note, that $s, t$ and $n$ forms an orthogonal system in both the left and right pictures of this figure.

The rotations can be expressed as the sum of change in slope of the middle surface and an additional average rotation due to shear effects:

$$
\vartheta_{s}=-\frac{\partial w_{0}}{\partial s}+\phi_{s}, \quad \vartheta_{t}=-\frac{1}{r} \frac{\partial w_{0}}{\partial \vartheta}+\phi_{t} .
$$

Here the terms due to shear effects are denoted by $\phi_{s}$ and $\phi_{t}$. The displacement vector at any point is:

$$
\{u\}=\left\{\begin{array}{lllll}
u_{0} & v_{0} & w_{0} & \vartheta_{s} & \vartheta_{t}
\end{array}\right\}^{T} .
$$

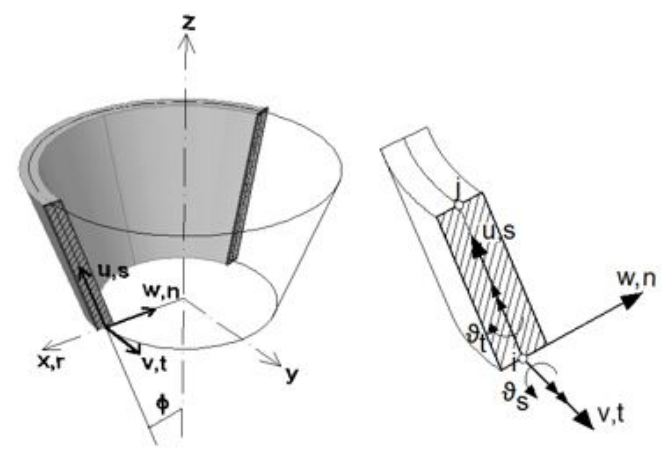

Fig. 2. Sign convention for displacements in a troncoconical shell

The elements of the small strain tensor in the local coordinate system $(s, t, n)$ are:

$$
\begin{gathered}
\varepsilon_{s}=\frac{\partial u}{\partial s}, \\
\varepsilon_{t}=\frac{1}{r} \frac{\partial v}{\partial \vartheta}+\frac{u}{r} \sin \phi-\frac{w}{R_{t}}, \\
\gamma_{s t}=\frac{1}{r} \frac{\partial u}{\partial \vartheta}+\frac{\partial v}{\partial s}-\frac{v}{r} \sin \phi-\frac{n}{R_{t}} \frac{\partial v}{\partial s}, \\
\gamma_{s n}=\vartheta_{s}+\frac{\partial w}{\partial s}, \\
\gamma_{t n}=\vartheta_{t}+\frac{1}{r} \frac{\partial w}{\partial \vartheta}+\frac{v}{R_{t}} .
\end{gathered}
$$

After substituting the expressions for the displacement field in the strain terms we can separate the strains due to membrane, bending and shear effects respectively, as:

$$
\left\{\varepsilon_{\text {membrane }}\right\}=\left\{\begin{array}{c}
\frac{\partial u_{0}}{\partial s} \\
\frac{1}{r} \frac{\partial v_{0}}{\partial \vartheta}+\frac{u_{0}}{r} \sin \phi-\frac{w_{0}}{r} \cos \phi \\
\frac{1}{r} \frac{\partial u_{0}}{\partial \vartheta}+\frac{\partial v_{0}}{\partial s}-\frac{v_{0}}{r} \sin \phi \\
0 \\
0
\end{array}\right\}
$$

$$
\begin{gathered}
\left\{\varepsilon_{\text {bending }}\right\}=\left\{\begin{array}{c}
\frac{\partial \vartheta_{s}}{\partial s} \\
\frac{1}{r} \frac{\partial \vartheta_{t}}{\partial \vartheta}+\frac{\vartheta_{s}}{r} \sin \phi \\
\frac{\partial \vartheta_{t}}{\partial s}+\frac{1}{r} \frac{\partial \vartheta_{s}}{\partial \vartheta}-\frac{\vartheta_{t}}{r} \sin \phi-\frac{\cos \phi}{r} \frac{\partial v_{0}}{\partial s}
\end{array}\right\}, \\
\left\{\varepsilon_{\text {shear }}\right\}=\left\{\begin{array}{c}
\vartheta_{s}+\frac{\partial w_{0}}{\partial s} \\
\frac{1}{r} \frac{\partial w_{0}}{\partial \vartheta}+\frac{v_{0}}{r} \cos \phi
\end{array}\right\},
\end{gathered}
$$

thus the strain vector can be composed as:

$$
\{\varepsilon\}=\left\{\varepsilon_{\text {membrane }}\right\}+\left\{\begin{array}{c}
n \cdot\left\{\varepsilon_{\text {bending }}\right\} \\
\left\{\varepsilon_{\text {shear }}\right\}
\end{array}\right\} .
$$


It has to be noted, that at the calculation of the shear contribution, the following assumptions have been made:

$$
1+\frac{n}{R_{t}}=1, \quad \frac{n^{2}}{R_{t}} \frac{\partial \vartheta_{t}}{\partial s}=0 \quad \text { and } \quad r=R_{t} \cos \phi
$$

\subsection{Stresses}

The stress resultants of a shell must have the same order as the generalized strains, therefore:

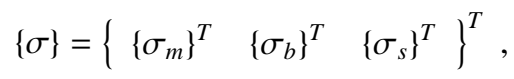

where,

$$
\begin{gathered}
\left\{\sigma_{m}\right\}=\left\{\begin{array}{lll}
N_{s} & N_{t} & N_{s t}
\end{array}\right\}^{T}, \\
\left\{\sigma_{b}\right\}=\left\{\begin{array}{lll}
M_{s} & M_{t} & M_{s t}
\end{array}\right\}^{T}, \\
\left\{\sigma_{s}\right\}=\left\{\begin{array}{ll}
Q_{s} & Q_{t}
\end{array}\right\}^{T},
\end{gathered}
$$

are the stress vectors due to membrane, bending and shear effects respectively. The sign convention is shown on Fig. 3 .

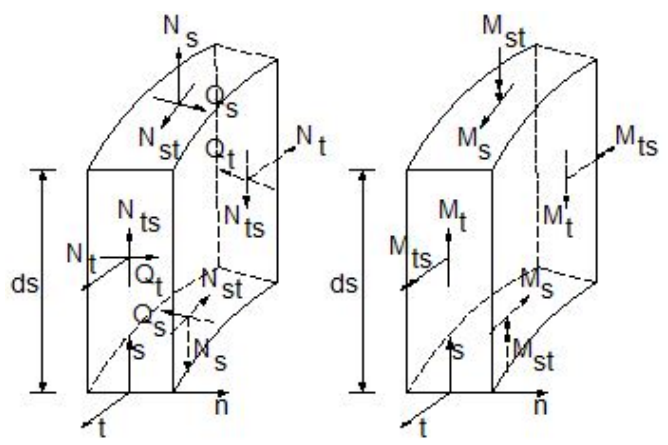

Fig. 3. Sign convention for stress resultants.

\subsection{Constitutive equations}

We applied Hooke's model do describe the connection between stresses and strains. It is only a first order approximation of the real material behavior, but it holds, if the forces and deformations are small enough. Therefore:

$$
\{\sigma\}=[D]\{\varepsilon\} \text {, where }[D]=\left[\begin{array}{ccc}
{\left[D_{m}\right]} & {[0]} & {[0]} \\
{[0]} & {\left[D_{b}\right]} & {[0]} \\
{[0]} & {[0]} & {\left[D_{s}\right]}
\end{array}\right]
$$

The sub - matrices are:

$$
\begin{aligned}
& {\left[D_{m}\right]=\left[\begin{array}{ccc}
1 & v & 0 \\
v & 1 & 0 \\
0 & 0 & \frac{1-v}{2}
\end{array}\right] \frac{E t}{\left(1-v^{2}\right)},} \\
& {\left[D_{b}\right]=\left[\begin{array}{ccc}
1 & v & 0 \\
v & 1 & 0 \\
0 & 0 & \frac{1-v}{2}
\end{array}\right] \frac{E t^{3}}{12\left(1-v^{2}\right)},} \\
& {\left[D_{s}\right]=K_{s}\left[\begin{array}{cc}
G t & 0 \\
0 & G t
\end{array}\right] .}
\end{aligned}
$$

\section{Finite strip formulation}

Because the steps of the formulation are basically identical to that for plates, a less detailed discussion should be satisfactory.

The displacement field within a strip can be approximated with the following expression by applying a summation over the number of nodes in the strip element $\left(i=1, \ldots, n_{e}\right)$ and over the number of Fourier terms $(l=1, \ldots, n)$ :

$$
\{u\}=\sum_{l=1}^{n} \sum_{i=1}^{n_{e}}\left[N_{i}^{l}\right]\left\{a_{i}^{l}\right\}
$$

where,

$$
\left[N_{i}^{l}\right]=\left[\begin{array}{ccccc}
N_{i} \cdot S_{l} & 0 & 0 & 0 & 0 \\
0 & N_{i} \cdot C_{l} & 0 & 0 & 0 \\
0 & 0 & N_{i} \cdot S_{l} & 0 & 0 \\
0 & 0 & 0 & N_{i} \cdot S_{l} & 0 \\
0 & 0 & 0 & 0 & N_{i} \cdot C_{l}
\end{array}\right]
$$

and

$$
\begin{gathered}
\left\{a_{i}^{l}\right\}=\left\{\begin{array}{lllll}
u_{0 i}^{l} & v_{0 i}^{l} & w_{0 i}^{l} & \vartheta_{s i}^{l} & \vartheta_{t i}^{l}
\end{array}\right\}^{T}, \\
S_{l}=\sin \left(\frac{l \pi}{\alpha} \vartheta\right) \quad \text { and } \quad C_{l}=\cos \left(\frac{l \pi}{\alpha} \vartheta\right) .
\end{gathered}
$$

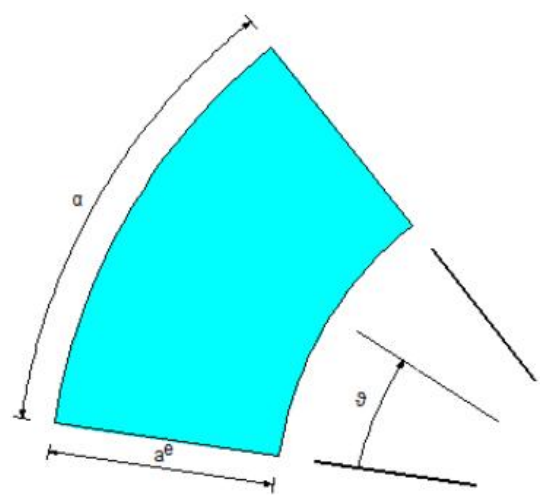

Fig. 4. Curved strip element

The meaning of the geometrical parameters is shown on Fig. 4. By having a look on the terms of the shape function matrix we can conclude, that the trigonometric expansion of the unknown displacements satisfies simply supported boundary conditions at $\vartheta=0$ and $\vartheta=\alpha$ with rigid diaphragms at the two ends.

The generalized strain vector has the form

$$
\{\varepsilon\}=\sum_{l=1}^{n} \sum_{i=1}^{n_{e}}\left[B_{i}^{l}\right]\left\{a_{i}^{l}\right\}
$$

where

$$
\left[B_{i}^{l}\right]=\left[\left[B_{m_{i}}^{l}\right]^{T} \quad\left[B_{b_{i}}^{l}\right]^{T} \quad\left[B_{s_{i}}^{l}\right]^{T}\right]^{T},
$$


with

$$
\begin{aligned}
& {\left[B_{m_{i}}^{l}\right]=} \\
& {\left[\begin{array}{ccccc}
\frac{\partial N_{i}}{\partial s} S_{l} & 0 & 0 & 0 & 0 \\
\frac{N_{i}}{r} \sin \phi S_{l} & -\frac{N_{i} l \pi}{b^{\prime}} S_{l} & -\frac{N_{i}}{r} \cos \phi S_{l} & 0 & 0 \\
\frac{N_{i} l \pi}{b^{\prime}} C_{l} & \left(\frac{\partial N_{i}}{\partial s}-\frac{N_{i}}{r} \sin \phi\right) C_{l} & 0 & 0 & 0
\end{array}\right]} \\
& {\left[\begin{array}{c}
B_{b_{i}}^{l}
\end{array}\right]=} \\
& {\left[\begin{array}{cccccc}
0 & 0 & 0 & \frac{\partial N_{i}}{\partial s} S_{l} & 0 \\
0 & 0 & 0 & \frac{N_{i}}{r} \sin \phi S_{l} & -\frac{N_{i} l \pi}{b^{\prime}} S_{l} \\
0 & -\frac{\partial N_{i}}{\partial s} \frac{\cos \phi}{r} C_{l} & 0 & \frac{N_{i} l \pi}{b^{\prime}} C_{l} & \left(\frac{\partial N_{i}}{\partial s}-\frac{N_{i}}{r} \sin \phi\right) C_{l}
\end{array}\right],} \\
& {\left[\begin{array}{l}
B_{s_{i}}^{l}
\end{array}\right]=\left[\begin{array}{ccccc}
0 & 0 & \frac{\partial N_{i}}{\partial s} S_{l} & N_{i} S_{l} & 0 \\
0 & \frac{N_{i}}{r} \cos \phi C_{l} & \frac{N_{i} l \pi}{b^{\prime}} C_{l} & 0 & N_{i} C_{l}
\end{array}\right]}
\end{aligned}
$$

Here $b^{\prime}=r \alpha$ and $\left[B_{m_{i}}^{l}\right],\left[B_{b_{i}}^{l}\right]$ and $\left[B_{s_{i}}^{l}\right]$ are the generalized strain matrices from membrane, bending and shear effects respectively, for node $i$ and the $l^{\text {th }}$ harmonic.

The expansion of the force vectors follow the same pattern as the displacements, therefore it is possible to write

$$
\left[\begin{array}{lll}
\{b\} & \{t\} & \{p\}
\end{array}\right]=\sum_{l=1}^{n}\left[\begin{array}{lll}
{\left[S_{l}\right]\{b\}^{l}} & {\left[S_{l}\right]\{t\}^{l}} & {\left[S_{l}\right]\{p\}^{l}}
\end{array}\right],
$$

where

$$
\left[S_{l}\right]=\left[\begin{array}{ccccc}
S_{l} & 0 & 0 & 0 & 0 \\
0 & C_{l} & 0 & 0 & 0 \\
0 & 0 & S_{l} & 0 & 0 \\
0 & 0 & 0 & S_{l} & 0 \\
0 & 0 & 0 & 0 & C_{l}
\end{array}\right]
$$

and $\{b\}^{l},\{b\}^{l}$ and $\{b\}^{l}$ are force amplitude vectors for the $l^{\text {th }}$ harmonic.

By the use of the formulation above the total potential energy of the shell can be created. Applying the stationary conditions the formulae for the stiffness matrix and the load vector of an element now becomes:

$$
\begin{aligned}
& {\left[K_{i j}^{l m}\right]^{e}=\left\{\begin{array}{ccc}
\frac{\alpha}{2} \int_{0}^{a_{e}}\left[\bar{B}_{i}\right]^{T}[D]\left[\bar{B}_{j}\right] r d s & \text { for } & l=m \\
0 & \text { for } & l \neq m
\end{array}\right. \text {, }} \\
& \left\{f_{i}^{l}\right\}=\iint_{A}\left[N_{i}^{l}\right]^{T}\{b\} d A+ \\
& +\iint_{A}\left[N_{i}^{l}\right]^{T}\{t\} d A+\iint_{A}\left[N_{i}^{l}\right]^{T}\{p\} d A .
\end{aligned}
$$

Matrix $\left[\bar{B}_{i}\right]$ can be simply obtained from $\left[B_{i}^{l}\right]$ by making $S_{l}=C_{l}=1$. The discretized equations of the system can be obtained by minimizing the Total Potential Energy of the shell with respect to all nodal amplitudes, which finally leads to an uncoupled system of equations, thus it can be solved separately for each harmonic.

\subsection{Coordinate transformation}

Contrary to plates, the strip elements of a folded plated structure meet in different angles, in other words they lie in different planes. Since all the variables of an element are expressed in its local coordinate system, it is necessary to transform the element arrays to a common, uniquely defined coordinate system. According to the theory, in the local coordinate systems only $\vartheta_{s}$ and $\vartheta_{t}$ is necessary to be defined. However, if considering the displacements from another system, all three rotation will have importance. If the axes of the global coordinate system are $\bar{X}, \bar{Y}$ and $\bar{Z}$, then it is possible to write that

$$
\left\{\bar{a}_{i}^{l}\right\}=[T]^{e}\left\{a_{i}^{l}\right\} \quad \text { and } \quad\left\{\bar{f}_{i}^{l}\right\}=[T]^{e}\left\{f_{i}^{l}\right\} \text {. }
$$

Here

$$
\left\{\bar{a}_{i}^{l}\right\}=\left\{\begin{array}{cccccc}
\bar{u}_{i}^{l} & \bar{v}_{i}^{l} & \bar{w}_{i}^{l} & \vartheta_{\bar{x}_{i}}^{l} & \vartheta_{\bar{y}_{i}}^{l} & \vartheta_{\bar{z}_{i}}^{l}
\end{array}\right\}^{T},
$$

and

$$
\left\{\bar{f}_{i}^{l}\right\}=\left\{\begin{array}{cccccc}
\bar{F}_{x_{i}}^{l} & \bar{F}_{y_{i}}^{l} & \bar{F}_{z_{i}}^{l} & M_{\vartheta_{\bar{x}_{i}}}^{l} & M_{\vartheta_{\bar{y}_{i}}}^{l} & M_{\vartheta_{\bar{z}_{i}}}^{l}
\end{array}\right\}^{T}
$$

are the generalized displacement and force vectors at node $i$ of element $e$ in the global coordinate system $\bar{X}, \bar{Y}, \bar{Z}$ where $\bar{Y}$ is parallel to $t$ and $\bar{Z}$ is the vertical axis, as it can be seen on Fig. 2. In expression (35) $[T]^{e}$ is the transformation matrix of the element. According to this thought, these vectors must be slightly modified in the local coordinate systems by adding a zero term, to facilitate the transformation, thus

$$
\left\{a_{i}^{l}\right\}=\left\{\begin{array}{llllll}
u_{0 i}^{l} & v_{0 i}^{l} & w_{0 i}^{l} & \vartheta_{s i}^{l} & \vartheta_{t i}^{l} & 0
\end{array}\right\}^{T},
$$

and

$$
\left\{f_{i}^{l}\right\}=\left\{\begin{array}{llllll}
F_{s_{i}}^{l} & F_{t_{i}}^{l} & F_{n_{i}}^{l} & M_{\vartheta_{s_{i}}}^{l} & M_{\vartheta_{t_{i}}}^{l} & 0
\end{array}\right\}^{T} .
$$

Then the stiffness matrix of an element for the $l^{\text {th }}$ term in the global coordinate system has the form

$$
\left[\bar{K}_{i j}^{l l}\right]^{e}=[T]^{e}\left[\hat{K}_{i j}^{l l}\right]^{e}[T]^{e^{T}},
$$

where

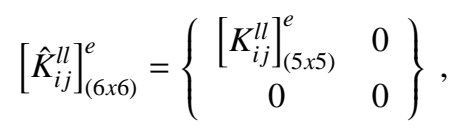

because the stiffness matrix has to be extended either to facilitate the transformation and to match the dimension of the extended versions of the other arrays of the element. Using expressions 20, and 26, the stiffness matrix can be written in a more practical form as

$$
\left[\bar{K}_{i j}^{l l}\right]^{e}=\frac{\alpha}{2} \int_{0}^{a_{e}}\left[\bar{B}_{i}^{*}\right]^{T}[D]\left[\bar{B}_{j}^{*}\right] r d s,
$$


where $\left[\bar{B}_{i}^{*}\right]$ can be obtained from $\left[\bar{B}_{i}\right]$ by means of the following transformation

$$
\left[\bar{B}_{i}^{*}\right]=\left[\bar{B}_{i}\right][T]^{e^{T}},
$$

which now allows the direct evaluation ofthe local stress resultants from the global displacements using Eq. 20). Thus the local stress resultants are:

$$
\{\sigma\}=\sum_{l=1}^{n} \sum_{i=1}^{n_{e}}[D]\left[B_{i}^{* l}\right]\left\{a_{i}^{l}\right\} .
$$

At a general folded plate, the matrix $\left[\bar{K}_{i j}^{l l}\right]^{e}$ will be fully populated. However, a problem arises if the strips meeting at a node lie in the same plane. In this situation, after the transformations the $6^{\text {th }}$ diagonal term of the element stiffness matrix with respect to the global coordinate system will be zero, thus the element stiffness matrix becomes singular, and the node is called a co-planar node. In the practice this singularity is avoided by putting an arbitrary value into this position of the matrix after the transformation. This solution implies, that this equation will be a pseudo equation. However, this step does not affect the solution process, since this equation is uncoupled from the other stiffness equations. This solution allows for all the coplanar and non-coplanar nodes to have the same number of degrees of freedom, which can be very useful if the solution system does not allow for varying numbers of variables at different nodes.

\section{Optimal design of folded plates}

The deterministic compliance design procedure of a linearly elastic 2D structure (disk) in plane stress with single loading is known from literature (e.g. Lógó [10]). This topology optimization problem extended to folded plates is given as follows:

$$
W=\sum_{g=1}^{G} \gamma_{g} l_{g} b_{g} t_{g}^{\frac{1}{p}}=\min !
$$

subject to

$$
\left\{\begin{aligned}
\mathbf{u}^{T} \mathbf{F}-C \leq 0 ; & \\
-t_{g}+t_{\min } \leq 0 ; & (\text { for } g=1, \ldots, G), \\
t_{g}-t_{\max } \leq 0 ; & (\text { for } g=1, \ldots, G) .
\end{aligned}\right.
$$

The value of the objective function $W$ means the total penalized volume of the structure in the function of the strip thicknesses, where the summation goes from one to the number of strips, denoted by $G$.

The strip element thicknesses $t_{g}$ are the design variables with lower bound $t_{\min }$ and upper bound $t_{\max }$, respectively. Furthermore $\gamma_{g}$ is the specific weight, $l_{g}$ and $b_{g}$ are the length and the width of the $g^{\text {th }}$ element. $\mathbf{u}^{T}$ is the displacement vector associated with the loading $\mathbf{F}$. The displacements $u$ can be calculated from $\mathbf{K u}=\mathbf{F}$, where $\mathbf{K}$ is the system stiffness matrix. $p$ is the penalty parameter $(p \geq 1)$ and the given compliance value is denoted by $C$ what depends on the displacement limit of the dedicated points. The above constrained mathematical programming problem can be solved by the use of an appropriate SIMP algorithm (Lógó [10]). The formulations above can lead to the same optimal solution as if the objective function and the compliance constraint were interchanged.

Another slight modification has to be evaluated if multiple loading and/or stochastic loading (Lógó [11,12]) is considered. Either the number of the compliance constraint is increased or the objective function 45 has to be modified to form a min-max problem. It can be happened as follow:

$$
\min \left[\max \mathbf{u}_{i}^{T} \mathbf{K} \mathbf{u}_{i}\right] i=1, \ldots, n,
$$

subject to

$$
\left\{\begin{array}{cl}
\sum_{g=1}^{G} \gamma_{g} l_{g} b_{g} t_{g}^{\frac{1}{p}}-W_{0} \leq 0 ; & \\
-t_{g}+t_{\min } \leq 0 ; & (\text { for } g=1, \ldots, G), \\
t_{g}-t_{\max } \leq 0 ; & (\text { for } g=1, \ldots, G) .
\end{array}\right.
$$

Here $n$ is the number of the independent load cases. $W_{0}$ is a given weight fraction of the structure. This type of problems can be solved by using the so called "parametric level" technique. Introducing a new parameter $C_{0}$ the min-max problem is substituted by a constrained minimization problem [5].

\subsection{Derivation of the optimality criteria formulation in case of single loading}

The necessary equations, which a thickness distribution has to satisfy to be the optimal solution, can be formulated with the so called Karush-Kuhn-Tucker (KKT) conditions. These conditions are derived from the slack variable approach and the classical technique of Lagrange multipliers. The difference to the classical approach is that the KKT conditions define the Lagrange multipliers to be sign definite while the Lagrange multiplier theorem only states the existence of them.

Therefore the conditions for a set $\left\{t_{1}, t_{2}, \ldots, t_{g}\right\}$ to be a local minimum of the objective function are the following:

$$
\begin{gathered}
\frac{\partial L}{\partial t_{g}}=\frac{1}{p} \gamma_{g} l_{g} b_{g} t_{g}^{\frac{1-p}{p}}-\lambda \mathbf{u}_{g}^{T} \frac{\partial \tilde{\mathbf{K}}_{g}}{\partial t_{g}} \mathbf{u}_{g}=0 ; g=1, \ldots, G, \\
\lambda \geq 0 ; \\
\mathbf{u}^{T} \mathbf{K u}-C \leq 0, \\
t_{\min } \leq t_{g}^{*} \leq t_{\max } ; g=1, \ldots n_{g}, \\
\lambda \cdot\left(\mathbf{u}^{T} \mathbf{K u}-C\right)=0,
\end{gathered}
$$

where $\frac{\partial \tilde{\mathbf{K}}_{g}}{\partial t_{g}}$ stands for the derivative of the strip stiffness matrix and $G$ denotes the number of strip elements. On the basis of 


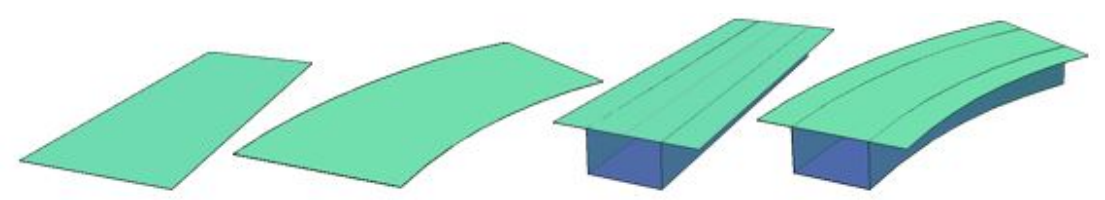

Fig. 5. Straight and curved rectangular plate and box-girder bridge
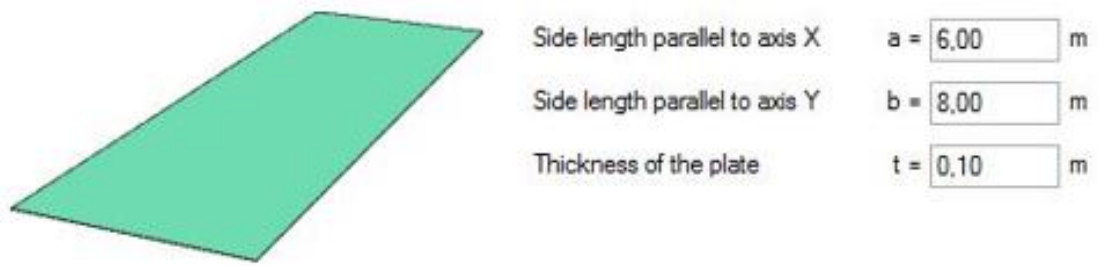

Fig. 6. Geometrical data of the first example

these equations an iterative formula can be derived, which leads to the optimal thickness distribution for one single load case.

$$
\begin{gathered}
t_{g}=\left(\frac{\lambda \cdot p \cdot R_{g}}{l_{g} \cdot b_{g}}\right)^{\frac{p}{1+p}}, \quad \text { where } \quad R_{g}=t_{g}^{2} \mathbf{u}_{g}^{T} \frac{\partial \tilde{K}_{g}}{\partial t_{g}} \mathbf{u}_{g}, \\
\lambda=\left(\frac{C}{C^{*}}\right)^{\frac{1+p}{p}}, \quad \text { where } \quad C^{*}=\left[\sum_{g}\left[\left(\frac{p \cdot R_{g}}{l_{g} \cdot b_{g}}\right)^{\frac{p}{1+p}} \mathbf{u}_{g}^{T} \hat{\mathbf{K}}_{g} \mathbf{u}_{g}\right]\right] .
\end{gathered}
$$

In expression 55 $\hat{\mathbf{K}}_{g}$ means $\frac{\partial \tilde{\mathbf{K}}_{g}}{\partial t_{g}} / t_{g}$. As usual, the box constraints are treated separately from the compliance inequality constraint, as they are not involved in the formulation of the Lagrangian function, but examined at every iteration cycle. Therefore, if a thickness happens to fall outside of the feasible set, in the next cycle it is forced to start with the boundary value $\left(t_{\max }\right.$ or $t_{\min }$ ) of the box.

\section{Numerical examples}

In the following, sample problems are introduced to illustrate the above explained methods. As already mentioned, the Finite Strip Method is used for the evaluation of the state variables, which excels in the calculation of structures with constant cross section. These include the following examples:

At each run, quadratic base functions were used in the cross section. The boundary conditions are also the same, according to the rules of the classical finite strip method (CFSM). This means that hinged supports were prescribed by choosing the proper trigonometric functions, while no additional boundary conditions were imposed.

\subsection{Straight rectangular plate}

Here we note, that in all cases, the cross sections should be understood in the x-z plane, therefore in Fig.5 size ' $a$ ' means the width of the plate and size 'b' means it's width and accordingly the thickness is the size in the ' $z$ ' direction.

The introduced plate was subjected to various loads, which positions are given with coordinates relative to the upper left corner, as seen in Fig. 7 .

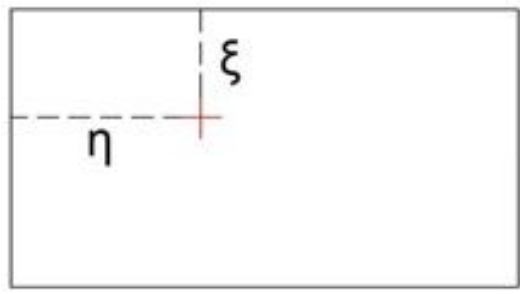

Fig. 7. Load coordinates for straight structures

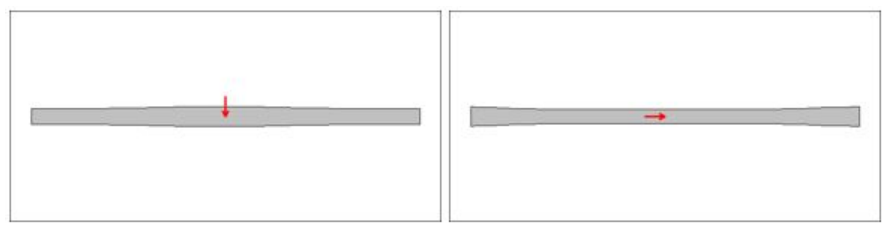

Fig. 8. Results for straight plates with $\boldsymbol{\xi}=3 \mathrm{~m}, \boldsymbol{\eta}=4 \mathrm{~m}$

The first load case is a single concentrated force $F=100 \mathrm{kN}$ pointing downwards, acting in the geometric middle of the structure, so according to the notation of Fig. 7, $\boldsymbol{\xi}=\mathbf{3} \mathbf{m}$ and $\boldsymbol{\eta}=\mathbf{4} \mathbf{m}$ in this case. The obtained thickness distribution can be seen on the left of Fig. 8 . The result for the same setup, but with a horizontal load is presented on the right of the same picture. With two concentrated forces of both $100 \mathrm{kN}$ placed at $\boldsymbol{\xi}=\mathbf{2} \mathbf{~ m}$ and $4 \mathrm{~m}, \eta=4 \mathrm{~m}$ :

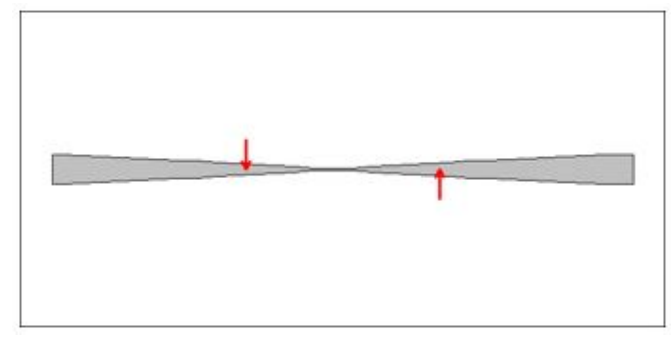

Fig. 9. Results for straight plates with $\boldsymbol{\xi}=2 \mathrm{~m}, \boldsymbol{\eta}=4 \mathrm{~m}$

\subsection{Curved rectangular plate}

The setup of these examples is the same as at the straight plate, with the difference, that the loads positions should be understood with the same value, but in a cylindrical relative coordinate system, illustrated on the next figure. 


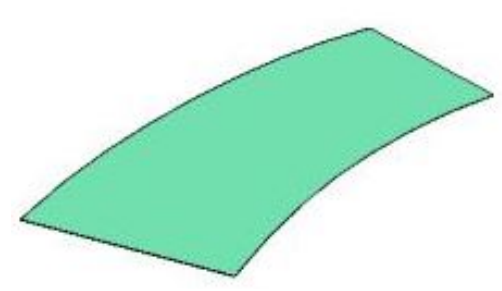

Width of the plate

$a=6.00 \quad \mathrm{~m}$

Curved length

$\mathrm{b}=8,00 \quad \mathrm{~m}$

Thickness of the plate

$\mathrm{t}=0.10 \quad \mathrm{~m}$

Radius

$\mathrm{R}=5.00 \mathrm{~m}$

Fig. 10. Geometrical data of the second example

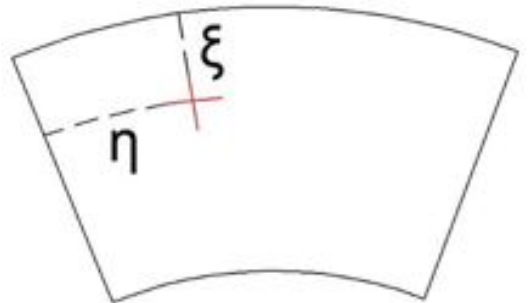

Fig. 11. Cylindrical load coordinates for curved structures

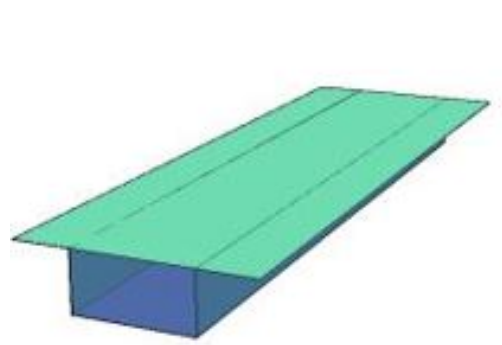

Width of the upper flange

af $1=6.00 \quad \mathrm{~m}$

Thickness of the upper flange

$t i 1=0,10 \quad \mathrm{~m}$

Width of the lower flange

$a \mathfrak{a}=3,00 \quad \mathrm{~m}$

Thickness of the lower flange

$\mathrm{t} 2=0.10 \mathrm{~m}$

Heigth of the webs

$a w=1.50 \quad \mathrm{~m}$

Thickness of the webs

$\mathrm{tw}=0,10 \quad \mathrm{~m}$

Side length parallel to axis $Y$

$b=10,00 \quad \mathrm{~m}$

Fig. 12. Geometrical data of the third example
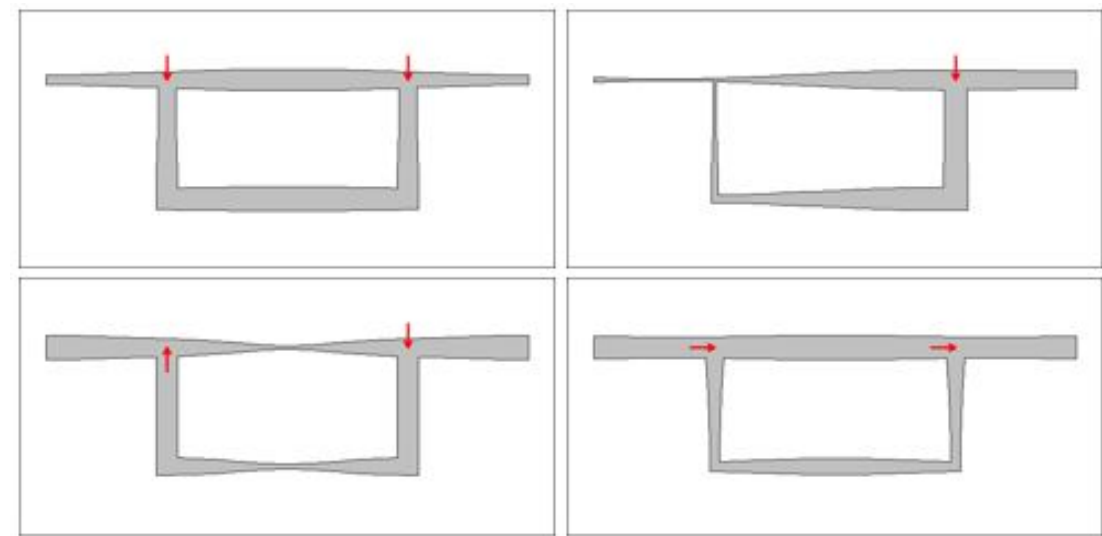

Fig. 13. Results for straight box-girder bridges

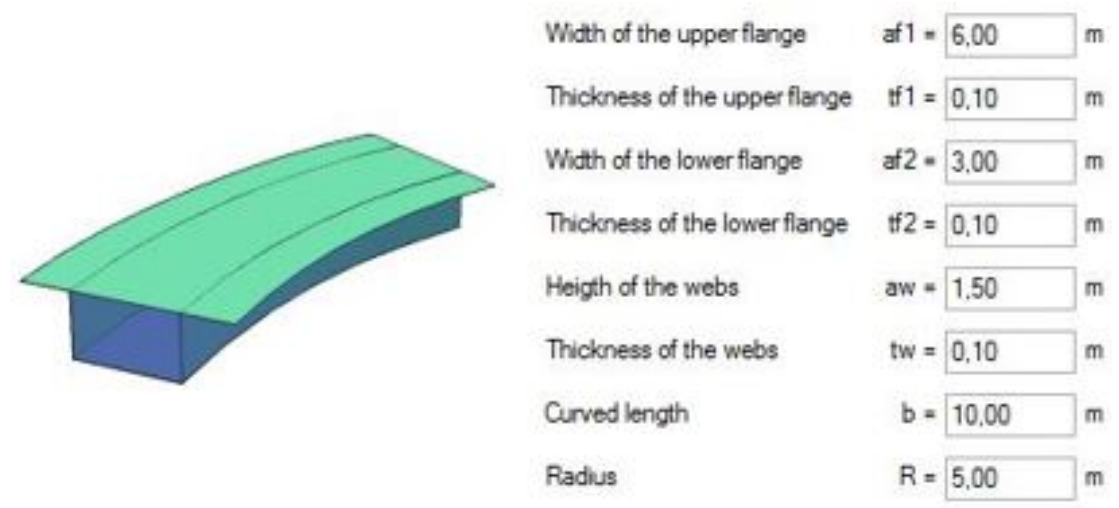

Fig. 14. Geometrical data of the fourth example 


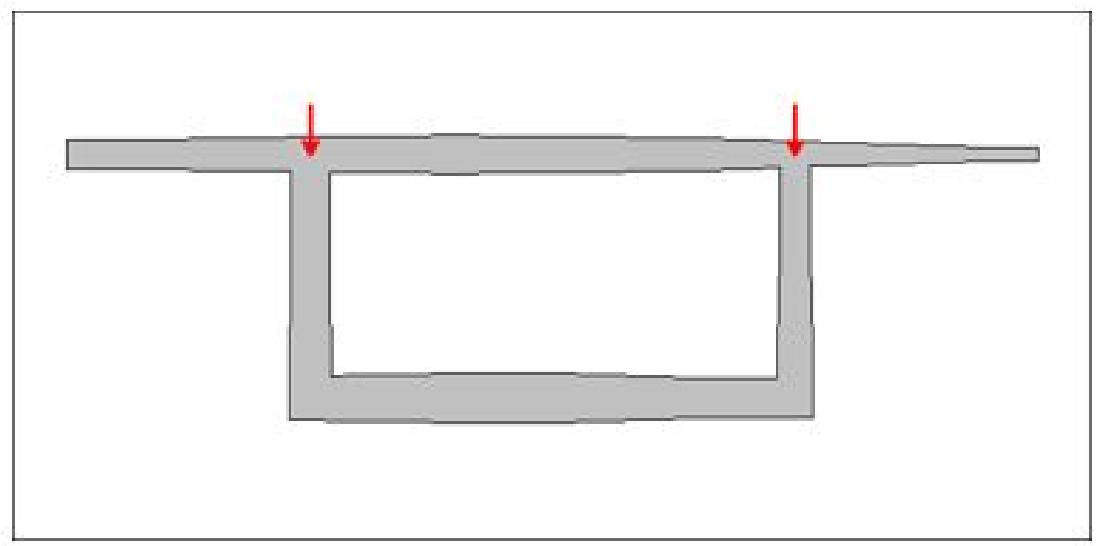

Fig. 15. Result for a curved box-girder bridge

\subsection{Straight box-girder bridge}

The geometry of this example is presented on Fig. 12 On the next figure (Fig. 13), the loads are always placed at the mid-span on the top flange at the position of the webs, so according to the notation of Fig. 11, $\boldsymbol{\xi}=\mathbf{2} \mathbf{~ m}$ and/or $\mathbf{4} \mathbf{~ m}, \boldsymbol{\eta}=\mathbf{5} \mathbf{~ m}$.

\subsection{Curved box-girder bridge}

The geometry of this example shows no difference to the previous one, except that the bridge has a curved geometry around the vertical axis. Only one load case was investigated, which can be put into comparison with the upper left picture of Fig. 13 The position of the concentrated forces should be understood as before.

\section{Conclusions}

A numerical procedure and computer program were elaborated for optimization of folded plates subjected to multiple loadings. The computational method is based on the finite strip method. The elaborated procedure with a slight modification can be suitable for the case of stochastic loading and/or multiple loading cases, as well. The surrogate loading system is problem dependent.

To make more appropriate models it is needed to make some additional investigations on the topic.

\section{Acknowledgement}

The present study was supported by the Hungarian National Scientific and Research Foundation (OTKA) (grant K 81185).

\section{References}

1 Balogh B, Lógó J, Optimal design of curved folded plates by optimality criteria method: Rodrigues HC, Herskovits J, Rodrigues CMM, Guedes JM, Araújo AL, Folgado JO, Moleiro F, Madeira JFA, Guedes JM (eds.), Engineering Optimization IV., CRC Press - Taylor and Francis Group; London, 2015, pp. 251-256.

2 Bergamini A, Biondini F, Finite strip modeling for optimal design of prestressed folded plate structures, Engineering Structures, 26(8), (2004), 10431054, DOI $10.1016 /$ j.engstruct.2004.03.005

3 Gellatly RA, Berke L, Optimality Criterion Based Algorithms: Gallagher RH, Zienkiewicz OC (eds.), Optimum Structural Design: Theory and Applications, Wiley; London, 1973, pp. 33-49.
4 Gilbert BP, Teh LH, Guan H, Self-shape optimisation principles: Optimisation of section capacity for thin-walled profiles, Thin-Walled Structures, 60, (2012), 190-204, DOI 10.1016/j.tws.2012.06.009

5 Kaliszky S, Lógó J, Application of Multicriteria Optimization in Layout Optimization of Structures: Jármai K, Farkas J (eds.), Metal Structures, Design, Fabrication, Economy, Millpress Science Publishers; Rotterdam, The Netherlands, 2003, pp. 271-276.

6 Lellep J, Optimal design of plastic reinforced cylindrical shells, Control Theory and Advanced Technology, 5, (1989), 119-135.

7 Lellep J, Paltsepp A, Optimization of Inelastic Cylindrical Shells with Stiffeners: Jármai $\mathbf{K}$, Farkas $\mathbf{J}$ (eds.), Design, Fabrication and Economy of Welded Structures, 2008, pp. 545-553, DOI 10.1533/9781782420484.13.545

8 Lellep J, Paltsepp A, Optimization of inelastic cylindrical shells with internal supports, Structural and Multidisciplinary Optimization, 41(6), (2010), 841-852, DOI $10.1007 / \mathrm{s} 00158-009-0465-2$

9 Leng J, Guest JK, Schafer BW, Shape optimization of cold-formed steel columns, Thin-Walled Structures, 49(12), (2011), 1492-1503, DOI 10.1016/j.tws.2011.07.009

10 Lógó J, New Type of Optimal Topologies by Iterative Method, Mechanics Based Design of Structures and Machines, 33(2), (2005), 149-171, DOI 10.1081/SME-200067035

11 Lógó J, New Type of Optimality Criteria Method in Case of Probabilistic Loading Conditions, Mechanics Based Design of Structures and Machines, 35(2), (2007), 147-162, DOI $10.1080 / 15397730701243066$

12 Lógó J, SIMP type topology optimization procedure considering uncertain load position, Periodica Polytechnica Civil Engineering, 56(2), (2012), 213219, DOI 10.3311/pp.ci.2012-2.07

13 Mroz Z, The Load Carrying Capacity and Minimum Weight Design of Annular Plates, Enginineering Transactions, 114, (1958), 605-625.

14 Prager W, Shield RT, Minimum Weight Design of Circular Plates under Arbitrary Loading, Zeitschrift für angewandte Mathematik und Physik ZAMP, 10(4), (1959), 421-426, DOI 10.1007/BF01601046

15 Rossow MP, Taylor JE, A Finite Element Method for the Optimal Design of Variable Thickness Sheets, AIAA Journal, 11(11), (1973), 1566-1569.

16 Rozvany GIN, Ong TG, Szeto WT, Sandler R, Olhoff N, Bendsvoe MP, Least-weight design of perforated elastic plates I-II., International Journal of Solids and Structures, 23(4), (1987), 521-536, 537-550, DOI $10.1016 / 0020$ 7683(87)90016-3 doi=10.1016/0020-7683(87)90015-1.

17 Shield RT, On the Optimum Design of Shells, Journal of Applied Mechanics, 27(2), (1960), 316-322, DOI $10.1115 / 1.3643959$ 